\title{
New approach in studies of microalgal cell lysis
}

Research Article

\author{
Maja Berden-Zrimec ${ }^{1 *}$, Jasmina Kožar Logar², Alexis Zrimec ${ }^{1}$, Luka Drinovec ${ }^{1}$ \\ Mladen Franko ${ }^{3}$, Alenka Malej ${ }^{4}$ \\ 'Institute of Physical Biology, \\ SI-1000 Ljubljana, Slovenia \\ 2"Jožef Stefan" Institute, \\ SI-1000 Ljubljana, Slovenia \\ ${ }^{3}$ University of Nova Gorica, \\ SI-5001 Nova Gorica, Slovenia
}

${ }^{4}$ National Institute of Biology, Marine Biology Station Piran, SI-6330 Piran, Slovenia

Received 25 November 2008; Accepted 10 March 2009

Abstract: A new approach to the studies of the microalgal cell lysis by utilizing a combination of two complementary methods is presented. Delayed fluorescence (DF) is a measure of the living algal biomass, detecting only cells with active photosynthesis. Thermal lens spectrometry (TLS) detects the total pigment amount released from lysed cells. Both methods select for photosynthetic organisms, reducing possible background from other sources (e.g. heterotrophic bacteria, zooplankton, and abiotic substances). The DF/TLS method was tested with a laboratory Skeletonema costatum culture exposed to a geometric dilution series of the lysing factor polyAPS. The exposure resulted in similar $\mathrm{EC}_{50}$ values for DF intensity, TLS and dissolved esterase activity of $0.8 \pm 0.2,1.77 \pm 0.35$, and $1.25 \pm 0.1 \mathrm{mg}$ poly-APS $\mathrm{I}^{-1}$, respectively. The combined DF/TLS method enabled a rapid evaluation of the living $v s$. dead cells without any sample pretreatment or manipulation.

Keywords: Phytoplankton • Cell lysis • Thermal lens spectrometry • Delayed fluorescence • Carotenoids • Photosynthesis

(c) Versita Warsaw and Springer-Verlag Berlin Heidelberg.

\section{Introduction}

The capacity to detect dead cells or cells with compromised membranes, and to estimate their proportion in phytoplankton communities is important for identification of the sources of dissolved organic carbon in natural pelagic communities [1]. Unfortunately, methods for quantification of such cells are scarce and determination of actual rates of phytoplankton lysis in the environment is problematic [2]. The biggest problem tends to be overestimation of the growth rate, because it is difficult to distinguish live cells from dead ones [3]. It has been suggested that automortality could be responsible for a significant reduction in population size [4] and should be considered the third major mortality factor next to grazing and sedimentation [3]. Riegman et al. estimated that at least $30 \%$ of biomass in phytoplankton blooms is lost by cell lysis [5].

Although cell death could be an important phytoplankton loss factor in some systems it can be negligible in others [6]. Therefore, good evaluation protocols are crucial for understanding the phytoplankton population dynamics. Several detection methods and subsequent interpretations have been proposed in attempt to quantify the cell lysis. Vital stains are frequently used to distinguish live from dead cells $[3,7,8]$. Some of these dyes are problematic because their emission spectrum is overlapping with that of the chlorophyll 
fluorescence [3]. In nature, distinguishing cells by using vital fluorescence stains is even more difficult due to the fluorescence background, ambiguities in establishing the threshold staining intensities and difficulties in identification of the stained cells [1]. Some techniques have been more successful, avoiding difficulties with the staining analyses. Agustí et al. have successfully tested the cell digestion assay, a non-staining membrane permeability test for quantification of living cells in nature $[1,6]$. Veldhuis et al. [3] have utilized a combination of autofluorescence measurements and DNA stains that do not have the same emission spectrum as chlorophyll $a$, and managed to distinguish three types of cells at different vital phases. Nevertheless, many errors can stem from sample manipulation. For example, incomplete chlorophyll a extraction can be one of the causes for interspecific variations in some studies [9].

In this paper, we present a similar approach to Stratton et al. [10], using a combination of complementary techniques: the thermal lens spectrometry (TLS) and delayed fluorescence (DF), to quantify cell lysis. Stratton et al. [10] presented an interesting approach in 1979, where they estimated cell lysis in Anabaena inaequalis. They plotted the decrease in optical density at $570 \mathrm{~nm}$ (which was linearly correlated to cell concentration) and the increase in absorbance of filtrates at $420 \mathrm{~nm}$ (which was linearly correlated to the cell lysis and pigment release). TLS detects released pigments from the lysed cells [11,12], whereas the DF measures the biomass of living algal cells [13-17]. Both methods are selective for photosynthetic organisms, thus reducing the problems with the background from other sources (e.g. heterotrophic bacteria, zooplankton, and abiotic substances) or autofluorescence [15]. Most importantly, the methods do not require any sample pretreatment or other manipulation before the measurement. The goal of this work was to test and evaluate the combination of TLS and DF techniques for monitoring of cell lysis by exposing the marine diatom Skeletonema costatum to a series of concentrations of a lysing substance under laboratory conditions.

\section{Experimental Procedures}

\subsection{Skeletonema costatum cultures}

The marine diatom Skeletonema costatum (Grev.) Cleve was isolated from the Gulf of Trieste by Alfred Beran (OGS, Trieste, Italy). The culture was maintained in $100 \mathrm{ml}$ Erlenmayer flasks containing $50 \mathrm{ml}$ of $\mathrm{f} / 2$ medium [18] at $20.5 \pm 0.5^{\circ} \mathrm{C}$, under cool-white fluorescent light (12:12 h light: dark cycle, average $50 \mu \mathrm{mol} \mathrm{m}^{-2} \mathrm{~s}^{-1}$ PPFD (Photosynthetic Photon Flux Density)).

\subsection{The cell lysis induction}

Cell lysis was induced in exponentially growing S. costatum cultures by addition of water soluble polymeric 3-alkyl pyridinium salts (poly-APS) with moderate cytotoxic and antimicrobial activity. Poly-APS was extracted from the marine sponge Reniera sarai [19]. Series of 10 poly-APS concentrations (dilution factor 2 ), ranging from 0.025 to $25 \mathrm{mg} \mathrm{l}^{-1}$ were used to induce the cell lysis in $50 \mathrm{ml} \mathrm{S}$. costatum cultures. Negative controls were cultures without added poly-APS. Cells were incubated in the poly-APS for 60 minutes.

\subsection{Thermal Lens Spectrometry (TLS)}

The controlled and lysed cultures were measured simultaneously with a double dual-beam thermal lens spectrometer (TLS), constructed especially for this purpose (Figure 1). Detailed description can be found in Kozar-Logar et al. [12]. TLS enabled realtime measurements [11,12], but for the purpose of this paper only the measurements after 60 minutes of incubation in PolyAPS were analyzed. In the TLS spectrometer, a peristaltic pump (Ismatec Sa, MS-4, Reglo/8) continuously pumps both samples through a closed loop system comprising of culture container and the TLS sample chamber [12]. A membrane filter $(0.22 \mu \mathrm{m}$ pore size) is inserted into each loop to prevent the cells from passing through the TLS sample chamber, so only the released contents from the lysed cells passes through. The TLS spectrometer consists of a relatively powerful Ar-laser, two $\mathrm{He}-\mathrm{Ne}$-lasers and photodiodes. The Ar-laser beam is divided into two equal beams. Each propagates through one TLS sample chamber. Released pigments in the water-flow absorb the Ar-laser light and induce a temperature rise of the water medium. This temperature rise changes the refractive index of the water medium which affects the propagation of the He-Ne laser beam through the TLS sample chamber a higher concentration of absorbing pigments results in a broader shape of the probe-beam and thus a higher intensity change on the photodiode.

The double dual-beam TLS is more sensitive for carotenoids than for chlorophylls, because the wavelength of the pump beam $(476.6 \mathrm{~nm})$ coincides with the absorption band of fucoxanthin and diadinoxanthin, the main carotenoids in $S$. costatum. In a previous study using HPLC chromatograms, it was proven that the absorbance of chlorophyll $a$ at this wavelength is negligible (chlorophylls represent less than $1 \%$ of the common peak area) [20]. The excitation of chlorophyll is therefore very small and chlorophyll fluorescence may diminish the conversion of the TLS signal to a very small extent. On the other hand, the fluorescence of carotenoids doesn't reduce the TLS signal. 


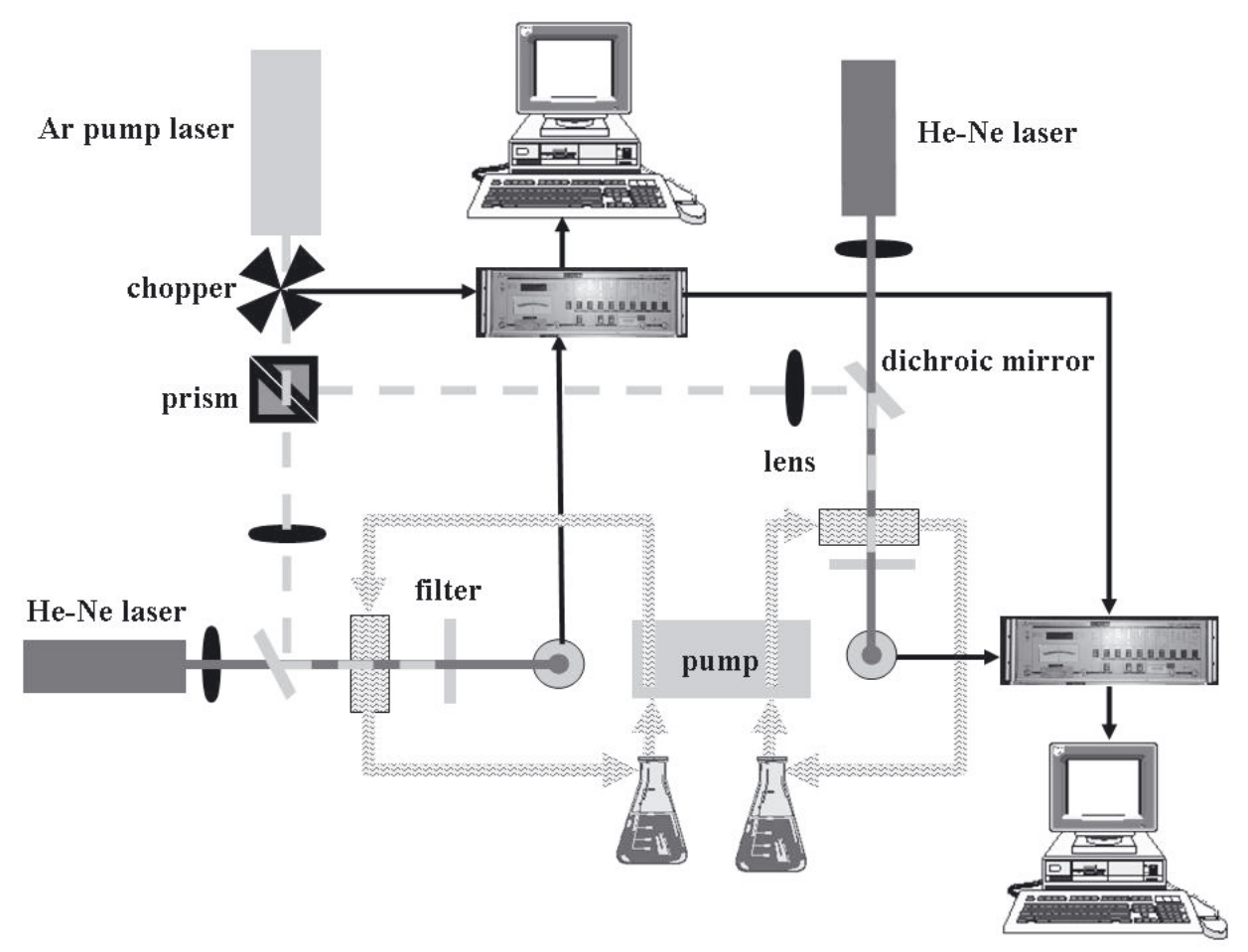

Figure 1. Schematics of the double dual-beam TLS spectrometer.

The $\beta$-carotene equivalent was introduced as a measure of pigments from the lysed phytoplankton. It is defined as the amount of an unknown mixture of pigments released by a lysed cell culture that would result in the same TLS signal as that from a known amount of $\beta$-carotene. The optimization, performance, calibration, determination of the limits of detection and sensitivity of the double dual-beam thermal lens spectrometer are fully described and discussed elsewhere [12]. The TLS values of $\beta$-carotene dilution series was compared with High Performance Liquid Chromatography (HPLC) [21].

\subsection{Delayed fluorescence intensity (DFI)}

DF was measured in a custom photon-counting luminometer with a red-light-sensitive photomultiplier tube (R1104, Hamamatsu) and a photon counting unit for signal conditioning and amplification (C3866, Hamamatsu) [22,23]. The samples for the DF measurement were pipetted from the cultures into $6 \mathrm{ml}$ round cuvettes (Sarstedt 58.485). DF of $2 \mathrm{ml}$ samples was measured before (the reference measurement) and 60 minutes after the addition of the poly-APS. The samples were incubated at $20.5 \pm 0.5^{\circ} \mathrm{C}$, under coolwhite fluorescent light (12:12 h light:dark cycle, average $50 \mu \mathrm{mol} \mathrm{m} \mathrm{s}^{-2} \mathrm{~s}^{-1}$ PPFD).
For DF measurements, the cuvettes were inserted in a thermostated sample holder $\left(21 \pm 0.1^{\circ} \mathrm{C}\right)$ of the luminometer. The samples were illuminated for $3 \mathrm{~s}$ with a $20 \mathrm{~W}$ halogen lamp $\left(60 \mu \mathrm{mol} \mathrm{m}^{-2} \mathrm{~s}^{-1}\right.$ PPFD at the sample position). DF was measured within a time interval of 1-60 s after the illumination. The background noise was measured prior to each DF measurement and subtracted from the data.

The delayed fluorescence intensity (DFI) was calculated as the average signal intensity in the measured time interval [22]. The resulting signal of the DFI inhibition induced by the poly-APS was calculated as a percentage of the control measurement (non-treated samples). DFI was calibrated with a geometric series of culture dilutions (factor 2) in order to determine living cell concentration in the samples exposed to the polyAPS. The cell concentrations of the measured samples were determined under a microscope (Neubauer Improved counting chamber), and used for the DFI signal calibration.

\subsection{Esterase activity (EA)}

The abundance of extracellular esterases was quantified from esterase activity at optimum temperature in the presence of excess substrate fluorescein diacetate (FDA) [3,24]. The esterase activity was measured on 
the same samples as DF intensity. After the second DF measurement (60 minutes after poly-APS addition), the samples were filtered through $0.45 \mu \mathrm{m}$ Milisart Sartorius filters. FDA (0.2 mM final concentration) was added to $1 \mathrm{ml}$ of the filtrate. Samples were incubated in the dark for 60 minutes at $20^{\circ} \mathrm{C}$. Fluorescence was measured in a custom fluorometer (IFB, Slovenia; Figure 2). A $450 \mathrm{~nm}$ high power LED (LED-450-01U, Roithner Lasertechnik, Austria) with a bandpass filter $447 \pm 30 \mathrm{~nm}$ (Semrock FF01-447/60-25, USA) was used for the sample excitation. Fluorescence was measured with a channel photomultiplier tube C1353P (Perkin Elmer, USA) in a photon counting mode with a bandpass filter $536 \pm 20 \mathrm{~nm}$ (Semrock FF01-536/40-25, USA) along with a shortpass filter 600 (Edmund optics, Techspec). The photomultiplier dark noise and background signal (the control) were subtracted from the measured data and the results were calculated as a percentage of fluorescence at maximal poly-APS concentration. The median effective concentration $\left(\mathrm{EC}_{50}\right)$ was defined as the polyAPS concentration that resulted in $50 \%$ inhibition of the parameter value compared to the control (non-treated cultures). $\mathrm{EC}_{50}$ of EA was compared to $\mathrm{EC}_{50}$ calculated from DFI and TLS measurements.

\section{Results and Discussion}

Concentrations of the poly-APS higher than $0.15 \mu \mathrm{g} \mathrm{ml}^{-1}$ initiated the cell lysis immediately after the addition of the lysing factor (measured with TLS, data not shown). TLS signal and DF intensity were compared 1 hour after the exposure to the poly-APS (Figure 3). Both TLS and DF were dependent on the poly-APS concentration. The dependence curves were sigmoidal (in semi-log plot) and typical for a dose-response relationship of a toxic action. The TLS signal increased with the poly-APS concentration, indicating that cell lysis was dependent on the release of intracellular carotenoids into the medium. The DF intensity (which is a measure of the living algal biomass) decreased with increasing polyAPS concentration. At the highest concentration of polyAPS, TLS increased for several hours then plateaued (data not shown). At lower poly-APS concentrations, cell lyses continued, although slowly, for at least seven hours (the time-span of TLS experiments). After 1 hour, the DF intensity was not yet maximally inhibited even at the highest poly-APS concentration (Figure 3). At this pre-selected time period, some of the cells were still alive - DFI yielded $17.6 \pm 3.6 \%$ of the nonexposed samples and TLS did not reach the maximum signal yet.

As can be seen from the Figure 3, TLS and DFI measurements are complementary. The sum of the TLS and DFI values was equal to the maximum at all polyAPS concentrations.

For the purpose of the evaluation of the living cell concentration after the cell lysis event, dependence of the DF intensity on the cell concentration in S. costatum was checked by a dilution series of full culture $\left(2 * 10^{6}\right.$ cells $\left.\mathrm{ml}^{-1}\right)$. The DF intensity was measured in a geometrical series of 10 cell concentrations (dilution factor 2), ranging from the culture density of approximately 200 to $2 * 10^{6}$ cells $\mathrm{ml}^{-1}$ (Figure 4a). The DF intensity was linearly correlated to the cell concentration (correlation 0.999, $\mathrm{P}<0.0001$ ) in the concentration range from 400 to $2 * 10^{6}$ cells $\mathrm{ml}^{-1}$. A similar linear relationship between DFI and cell concentration was found in the marine dinoflagellate Prorocentrum minimum [22]. Below the concentration

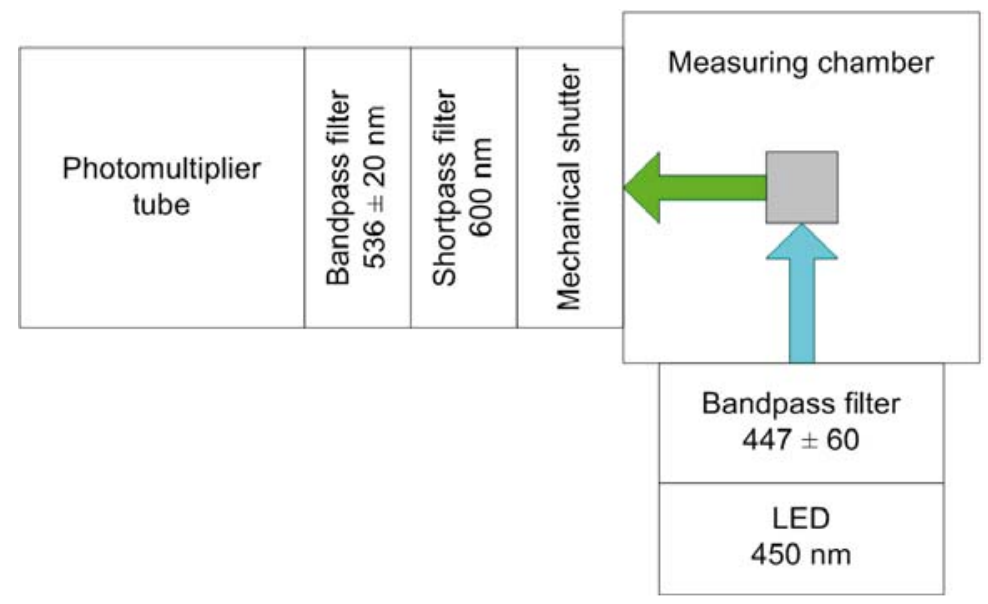

Figure 2. The fluorometer setup for measuring fluorescein. 


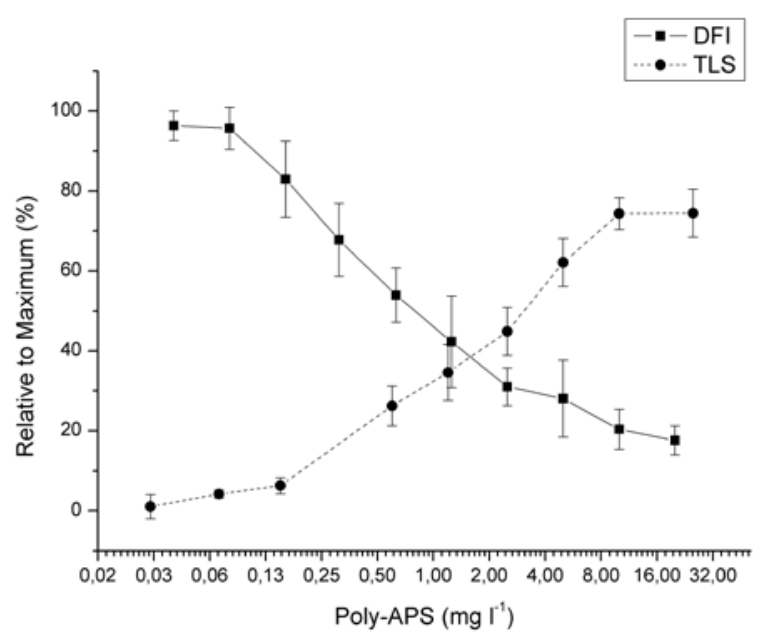

Figure 3. TLS (circles) and DFI (squares) of Skeletonema costatum after a $1 \mathrm{~h}$ incubation in a cell lysing substance (poly-APS), represented as percent of maximum TLS or DFI value. For TLS, the maximum value was defined as the signal at the highest poly-APS concentration after a 7 hour incubation and for DFI, as the signal of the non-treated sample. Error bars represent standard deviations of three replicates for TLS and eight replicates for DFI.

of 400 cells $\mathrm{ml}^{-1}$, the luminometer could no longer distinguish changes in the biomass as measured by DFI. From the measured calibration curve (Figure 4a), the concentration of living cells with active photosynthesis was evaluated. Similarly, the concentration of lysed cells was evaluated from TLS signal calibration curve (Figure 4b).

The DF intensity was compared to the esterase activity method measured on the same samples (Figure 5). The response of $S$. costatum culture to a geometric series of poly-APS dilutions was different from Figure 3, probably because a different culture was used. The effective concentration of poly-APS $\left(\mathrm{EC}_{50}\right.$, concentration that resulted in $50 \%$ of the maximum signal), was slightly different for the three techniques: $1.25 \pm 0.1 \mathrm{mg} \mathrm{l}^{-1}$ for the dissolved esterase activity (EA) and $0.3 \pm 0.1 \mathrm{mg} \mathrm{l}^{-1}$ for DFI in one culture of $S$. costatum (Figure 5), and $1.77 \pm 0.35 \mathrm{mg} \mathrm{l}^{-1}$ for TLS and $0.8 \pm 0.2 \mathrm{mg} \mathrm{l}^{-1}$ for DFI in a different culture (Figure 3).

TLS and DFI represent two complementary approaches to monitoring cell lysis. TLS measures the concentration of pigments released from damaged photosynthetic cells, whereas DFI detects living algal biomass. The advantage of both methods is that they do not require any sample preparation and enable real time monitoring of algal population dynamics. Both methods are selective for photosynthetic organisms, thus reducing possible background noise from other sources (e.g. heterotrophic bacteria, zooplankton, and abiotic substances).

TLS is a unique technique which enables monitoring the cell lysis dynamic processes in real time by non- selective detection of the total pigment amount released from cells into the growth media. It is extremely sensitive and can detect as low as $30 \mathrm{fg}$ of carotenoids per $\mathrm{ml}$ in organic solvents, but less in the seawater ( $0.6 \mathrm{ng}$ per $\mathrm{ml}$ ) $[20,25]$. In this experiment, the TLS real-time method enabled detection of $6 \cdot 10^{3}$ lysed $S$. costatum cells $\mathrm{ml}^{-1}$. The limit of detection and sensitivity could be lowered by at least a factor of 100 in stationary measurements, though. The sensitivity of DF intensity in this study was no more than several 100 living cells $\mathrm{ml}^{-1}$, but a higher sensitivity could be achieved by using bigger sample volumes and a detection system, which enables higher detection of delayed fluorescence light (Montecino et al., submitted for publication). An additional advantage of the delayed fluorescence technique is that it can distinguish among several algal taxonomic groups by using DF excitation spectra $[15,26]$. For example, monitoring changes in phytoplankton composition during growth enables better analysis and interpretation of the measured data.

The combined TLS/DF technique is suitable for laboratory measurements of cell lysis provoked by different environmental factors (e.g. presence of harmful substances, suboptimal growth conditions, nutrient and light limitations). In the case of laboratory monocultures, the amount of lysed cells over time can be calculated by calibrating DFI by geometric dilutions of the experimental cell culture. In field studies, DFI could only be used to monitor living phytoplankton biomass changes if we knew the initial biomass before any population decline due to cell lysis (at the beginning of the phytoplankton bloom, etc.). On the other hand, TLS can be calibrated to measure absolute carotenoid concentrations in 

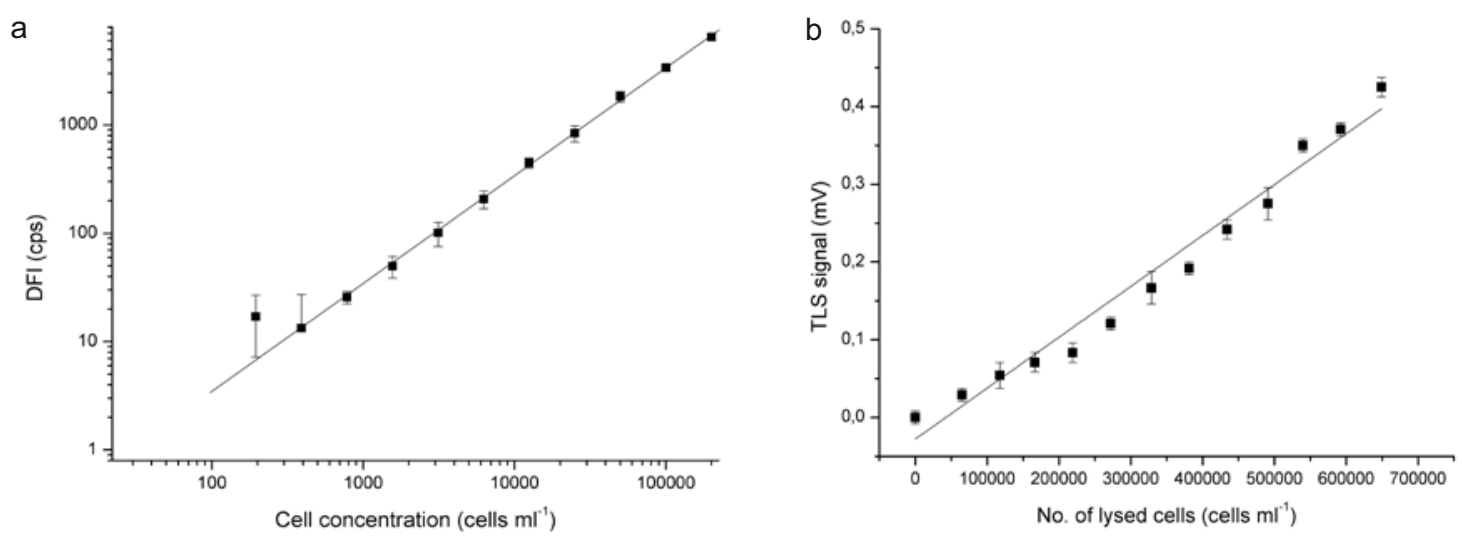

Figure 4. DFI (a) and TLS signal (b) as a function of the cell concentration. The line represents a linear fit of the data, given by $Y=A+B * X$. The correlation coefficients ( $R^{2}$ values) were 0.999 and 0.97 for DFI and TLS, respectively. Error bars represent standard deviations of three replicates, $\mathrm{cps}=$ counts per second.

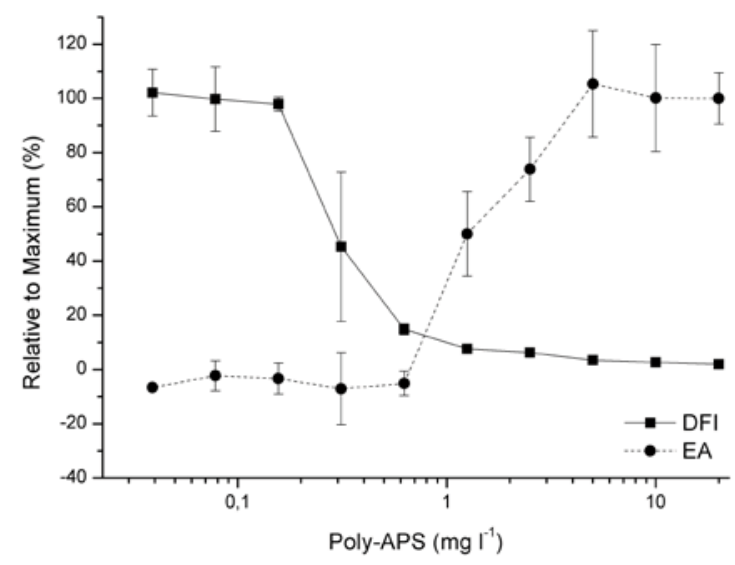

Figure 5. Esterase activity (EA) and DF intensity (DFI) of the same samples of Skeletonema costatum after a $1 \mathrm{~h}$ incubation in a cell lysing substance (poly-APS), represented as percent of maximum. Error bars represent standard deviations of three replicates.

environmental samples. If the pigment (e.g. carotenoid) decomposition rate were known, one could use TLS measurements of the dissolved pigment content to determine the algal biomass that lysed over a certain time interval. If the assumption that the carotenoid background is known and can be actually determined by combination of HPLC - TLS measurements was correct, the cell lysis rate (i.e. percent of lysed biomass in a certain time interval) could thus be determined from a measurement performed on a single sample. Furthermore, by comparing the combination of normalized DFI and TLS data to maximal values and calibrating to cell concentration, one could calculate the percentage of cells lysed in a single sample as the quotient TLS/(DFI+TLS).

\section{Acknowledgements}

This work was co-financed by the Slovenian Research Agency (grant \#P1-0237). We are thankful to Alfred Beran for providing the Skeletonema culture, to Kristina Sepcic for the poly-APS and Lidija Berden for revising the language. 
[1] Agusti S., Sanchez M.C., Cell viability in natural phytoplankton communities quantified by a membrane permeability probe, Limnol. Oceanogr., 2002, 47, 818-828

[2] Berman T., Wynne D., Assessing phytoplankton lysis in Lake Kinneret, Limnol. Oceanogr., 2005, 50, 526537

[3] Veldhuis M.J.W., Kraay G.W., Timmermans K.R., Cell death in phytoplankton: correlation between changes in membrane permeability, photosynthetic activity, pigmentation and growth, Eur. J. Phycol., 2001, 36, 167-177

[4] Berges J.A., Falkowski P.G., Physiological stress and cell death in marine phytoplankton: Induction of proteases in response to nitrogen or light limitation, Limnol. Oceanogr., 1998, 43, 129-135

[5] Riegman R., Van Bleijswijk J.D.L., Brussaard C.P.D., The use of dissolved esterase activity as a tracer of phytoplankton lysis - Comment, Limnol. Oceanogr., 2002, 47, 916-920

[6] Agusti S., Alou E., Hoyer M.V., Frazer T.K., Canfield D.E., Cell death in lake phytoplankton communities, Freshw. Biol., 2006, 51, 1496-1506

[7] Baldi F., Minacci A., Saliot A., Mejanelle L., Mozetic P., Turk V., et al., Cell lysis and release of particulate polysaccharides in extensive marine mucilage assessed by lipid biomarkers and molecular probes, Mar. Ecol. - Progr. Ser., 1997, 153, 45-57

[8] van Boekel W.H.M., Hansen F.C., Riegman R., Bak R.P.M., Lysis-induced decline of a Phaeocystis spring bloom and coupling with the microbial foodweb, Mar. Ecol. - Progr. Ser., 1992, 81, 269-276

[9] Agusti S., Duarte C.M., Addressing uncertainties in the assessment of phytoplankton lysis rates in the sea - Comment, Limnol. Oceanogr., 2002, 47, 921924

[10] Stratton G.W., Huber A.L., Corke C.T., Effect of mercuric ion on the growth, photosynthesis, and nitrogenase activity of Anabaena inaequalis, Appl. Environ. Microbiol., 1979, 38, 537-543

[11] Kozar-Logar J., Malej A., Franko M., On-line monitoring of diatom lysis by thermal lens spectrometry, J. Phys. IV, 2005, 125, 705-707

[12] Kozar-Logar J., Malej A., Franko M., Double dual beam thermal lens spectrometer for monitoring of phytoplankton cell lysis, Instrum. Sci. Technolog., 2006, 34, 23-31

[13] Berden-Zrimec M., Drinovec L., Zrimec A., Tisler T., Delayed fluorescence in algal growth inhibition tests, Cent. Eur. J. Biol., 2007, 2, 169-181
[14] Katsumata M., Koike T., Nishikawa M., Kazumura K., Tsuchiya H., Rapid ecotoxicological bioassay using delayed fluorescence in the green alga Pseudokirchneriella subcapitata, Water Res., 2006, 40, 3393-3400

[15] Istvanovics V., Honti M., Osztoics A., Shafik H.M., Padisak J., Yacobi Y., et al., Continuous monitoring of phytoplankton dynamics in Lake Balaton (Hungary) using on-line delayed fluorescence excitation spectroscopy, Freshw. Biol., 2005, 50, 1950-1970

[16] Strehler B.L., Arnold W., Light production by green plants, J. Gen. Physiol., 1951, 34, 809-820

[17] Wiltshire K.H., Harsdorf S., Smidt B., Blocker G., Reuter R., Schroeder F., The determination of algal biomass (as chlorophyll) in suspended matter from the Elbe estuary and the German Bight:Acomparison of high-performance liquid chromatography, delayed fluorescence and prompt fluorescence methods, J. Exp. Mar. Biol. Ecol., 1998, 222, 113-131

[18] Guillard R.R.L., Culture of phytoplankton for feeding marine invertebrates, In: Smith W.L., Chanley M.H. (Eds.), Culture of marine invertebrate animals, Plenum Press, New York, 1975

[19] Sepcic K., Batista U., Vacelet J., Macek P., Turk T., Biological activities of aqueous extracts from marine sponges and cytotoxic effects of 3-alkylpyridinium polymers from Reniera sarai, Comp. Biochem. Physiol. C, Comp. Pharmacol. Toxicol., 1997, 117, 47-53

[20] Kozar-Logar J., Development of laser spectroscopic techniques for characterisation and studies of phytoplankton pigments, $\mathrm{PhD}$ thesis, Polytechnic, School of Environmental Sciences, Nova Gorica, Slovenia, 2003

[21] Jeffrey S.W., Mantoura R.F.C., Development of pigment methods for oceanography: SCOR supported working groups and objectives, In: Jeffrey S.W., Mantoura R.F.C., Wright S.W. (Eds.), Phytoplankton pigments in oceanography: guidelines to modern methods, UNESCO Publishing, Paris, 1997

[22] Monti M., Zrimec A., Beran A., Berden-Zrimec M., Drinovec L., Kosi G., et al., Delayed luminescence of Prorocentrum minimum under controlled conditions, Harmful Algae, 2005, 4, 643-650

[23] Zrimec A., Drinovec L., Berden-Zrimec M., Influence of chemical and physical factors on longterm delayed fluorescence in Dunaliella tertiolecta, Electromagn. Biol. Med., 2005, 24, 309-318 
[24] Agusti S., Satta M.P., Mura M.P., Benavent E., Dissolved esterase activity as a tracer of phytoplankton lysis: Evidence of high phytoplankton lysis rates in the northwestern Mediterranean, Limnol. Oceanogr., 1998, 43, 1836-1849

[25] Luterotti S., Franko M., Sikovec M., Bicanic D., Ultrasensitive assays of trans- and cis-betacarotenes in vegetable oils by high-performance liquid chromatography- thermal lens detection, Anal. Chim. Acta, 2002, 460, 193-200
[26] Yacobi Y.Z., Gerhardt V., Gonen-Zurgil Y., Sukenik A., Delayed fluorescence excitation spectroscopy: A rapid method for qualitative and quantitative assessment of natural population of phytoplankton, Water Res., 1998, 32, 2577-2582 